\title{
Sur les traces du " problématique voyageur »
}

\section{Damien Zanone}

\section{OpenEdition}

\section{Journals}

Édition électronique

URL : http://journals.openedition.org/recherchestravaux/165

DOI : 10.4000/recherchestravaux.165

ISSN : 1969-6434

\section{Éditeur}

UGA Éditions/Université Grenoble Alpes

\section{Édition imprimée}

Date de publication : 15 avril 2007

Pagination : 5-10

ISBN : 978-2-84310-107-7

ISSN : 0151-1874

\section{Référence électronique}

Damien Zanone, «Sur les traces du «problématique voyageur » ", Recherches \& Travaux [En ligne], 70 2007, mis en ligne le 26 novembre 2008, consulté le 23 septembre 2020. URL : http://

journals.openedition.org/recherchestravaux/165; DOI : https://doi.org/10.4000/recherchestravaux 165

(C) Recherches \& Travaux 
Damien ZANONE

Université Stendhal-Grenoble 3

\section{Présentation Sur la trace du " problématique voyageur "}

À Henri Bonnet.

Depuis plus de trente ans que George Sand a retrouvé une postérité active, que ses œuvres renaissent à l'édition et suscitent à nouveau le commentaire, la situation des Lettres d'un voyageur ne laisse pas d'être paradoxale. Quand, en 197I, l'ouvrage fut remis en circulation par deux rééditions simultanées (l'une établie par Georges Lubin, l'autre par Henri Bonnet ${ }^{\mathrm{I}}$ ), il prenait place comme un élément marquant de la résurrection critique de l'auteur. Il n'est pas de "sandien " depuis lors qui, d'accord avec tous ceux que séduit la parole romantique, ne reconnaisse à ce recueil de douze " lettres " une place de premier ordre dans la production de Sand comme dans l'invention d'une prose lyrique. Et pourtant l'ouvrage continue à intimider l'analyse : alors que, de George Sand, le continent romanesque et la masse des écrits intimes (lettres personnelles et autobiographie) sont parcourus de part en part, les très singulières Lettres d'un voyageur ont relativement peu suscité l'étude².

I. Ces éditions restent les éditions de référence auxquelles il sera renvoyé pour toutes les citations du présent volume; pages indiquées entre parenthèses et dans l'ordre suivant : G. Sand, Lettres d'un voyageur, in Euvres autobiographiques, édition de G. Lubin, Paris, Gallimard "Bibliothèque de la Pléiade ", vol. II, I971, p. 633-943 ; G. Sand, Lettres d'un voyageur, édition de $\mathrm{H}$. Bonnet, Paris, Flammarion "GF », I97I (réédité en 2004 avec une bibliographie actualisée).

2. La bibliographie critique rassemblée en fin de volume permet de prendre la mesure exacte de ces travaux (à la date de 2007). 
C'est comme si, à force d'être trop divers dans ses thèmes et trop instable dans sa forme, le texte n'autorisait que de libres lectures, restant rebelle au discours critique et le défiant d'imposer ses normes. Chacun s'accorde à saluer la richesse des Lettres d'un voyageur et à admirer, comme Henri Bonnet, qu' " elles rassemblent en gerbe tout ce que le romantisme a été, sentimental, politique, poétique, mystique, musical, etc. ${ }^{3}$; chacun subit la séduction de ce recueil imprévisible qu'il est tentant de lire, en suivant la suggestion de Nicole Mozet, comme un "véritable traité de poétique sandienne et romantique ${ }^{4} »$.

Réunis à Grenoble pendant deux jours de juin 2004, la quinzaine de chercheurs dont les articles sont rassemblés ci-après ont voulu relever le défi de ces propositions. Invités à soumettre les Lettres d'un voyageur à une observation et à un commentaire systématiques, ils ont eu le souci de comprendre l'attrait que le recueil exerce, l'embarras qu'il provoque parfois, en interrogeant ses principaux aspects : par exemple, les perturbations d'une énonciation changeante (entre presse et livre, entre masculin et féminin, entre destinataires divers) ; la variation continue des registres d'une parole tour à tour intime, lyrique, facétieuse, descriptive et polémique ; l'affranchissement visà-vis des genres codifiés (le récit de voyage, la confession autobiographique, l'article polémique, le compte rendu musical...) ; l'instabilité thématique produite, au fil de cette poétique ouverte, par la quête d'expression de soi et le dialogue avec les autres arts.

Sur ces pistes, et prêt à en voir surgir d'autres encore, chacun s'est mis sur la trace du " problématique voyageur " qu'avoue George Sand en préface à la deuxième édition de son œuvre (p. 647 ; p. $39^{5}$ ). Où va ce voyageur et que dit-il ? Le sens et la cohérence de son parcours ne sont pas donnés d'emblée, ni jamais explicitement nommés, dans ces douze « Lettres » dont les aspects d'abord perçus sont la diversité et l'instabilité. Dans ces conditions, inévitablement, la lecture critique tend à chercher l'unité du recueil, n'acceptant de suivre le voyageur-narrateur dans ses mille et un détours que pour mieux l'attraper enfin et lui faire dire qui il est et ce qu'il veut. L'unité du recueil, c'est le mirage qui attire toutes les lectures et qu'aucune ne désespère de fixer, c'est une construction qui est autant dans le livre que dans son lecteur, c'est

3. H. Bonnet, «Introduction ", in G. Sand, Lettres d'un voyageur, édition de H. Bonnet, op. cit., p. 22.

4. N. Mozet, George Sand, écrivain de romans, Saint-Cyr-sur-Loire, Christian Pirot éditeur, 1997, p. 9.

5. Selon la convention que nous adoptons pour ce volume: G. Sand, Lettres d'un voyageur, pages de l'édition de G. Lubin puis pages de l'édition de H. Bonnet. 
le motif dans le tapis dont chacun espère qu'il dessinera une clé donnant accès au secret de la littérature. Les Lettres d'un voyageur ont ce mérite immense d'obliger chaque lecteur, vis-à-vis d'elles, à problématiser son rapport à la littérature, à s'interroger sur ce qu'il y cherche et à éprouver ses velléités herméneutiques comme autant de tentatives audacieuses et fragiles.

Penser et dire l'unité d'un recueil qui garantit si peu d'en avoir apparaît ainsi comme un leitmotiv insistant dans les pages qui vont suivre, la plupart des articles s'attachant à saisir l'ouvrage dans son entier. L'audace et la fragilité de la démarche étant reconnues, on peut essayer de dégager quelques lignes de force que mettent en lumière ces travaux par leurs éclairages convergents sur la même œuvre : le débat conflictuel entre l'art et la vie, le départ vers la fiction, la part faite au lecteur.

Dans les Lettres d'un voyageur, les formes canoniques de l'art sont malmenées et finalement rejetées, le Voyageur montrant une fougue iconoclaste à les fouler aux pieds : afin de mieux faire sa place à l'artiste, afin de lui donner un espace neuf où inventer. "Meurtre symbolique de l'art $^{6}$ ": les statues redeviennent de misérables pierres, le grand opéra est délaissé au profit de la musique intérieure, la littérature est réinventée au plus près de soi, dans l'inquiétude sans doute, c'est-à-dire dans la vie. Répudier les formes du passé revient à dire que l'art est devant soi, pas derrière.

Dans l'entre-deux formel où se complaisent les "Lettres ", dans cette indétermination qu'elles construisent, scintille et surgit la fiction comme réponse et pied de nez aux codes, aux savoirs positifs. Les Lettres d'un voyageur créent cet espace où dire « je » devient possible, où le « je " s'extrait du " nous " dont il reste pourtant solidaire. Aux dernières lignes de la dernière lettre, l'affirmation de "George Sand et compagnie " proclame l'appartenance à la confrérie des artistes en même temps que le pseudonyme définitivement conquis, qui est identité singulière. Voyageur, en mouvement et en devenir, ce « je » se déplace en sachant toujours où est la ligne de fuite, l'horizon, "cette patrie des âmes inquiètes " $(875 ; 254)$. C'est ce battement entre un ici et un ailleurs que dit le voyage, c'est là qu'existe la fiction, c'est là que trouve place "George Sand » et qu'elle nous invite à la rejoindre.

Le lecteur, en effet, n'est pas laissé sur le bord du chemin. Le sentiment de liberté ordonnée que produit le recueil est valable comme sentiment de soi ; chaque lecteur peut s'emparer de la figuration de soi que propose l'auteur, comme chambre d'échos où se repérer parmi les voix et les discours du

6. N. Savy, «Canova, le marbre et la montagne : une poétique de la pierre dans les Lettres d'un voyageur", p. I8I du présent volume. 
monde. Pour dialoguer avec le "problématique voyageur ", arrêter un instant sa course et entendre son expérience, il faut se faire soi-même problématique lecteur : prêt à voyager dans le texte et à l'accueillir en soi, lui trouver, le temps de la lecture, unité, sans prétendre sceller de quelque formule. Lire les Lettres d'un voyageur, c'est contempler la liberté ordonnée en soi.

Cette lecture, le présent volume propose de la faire en se penchant d'abord sur les questions que suscite un texte au genre si incertain : Béatrice Didier se demande s'il faut y voir la création d'un genre, Marie-Ėve Thérenty mesure les effets du passage de l'édition en revue à celle en recueil, Brigitte Diaz évalue les « Lettres » en tant que lettres, Martine Reid questionne leur énonciation et Françoise Genevray leurs dispositifs d'adresse.

Les contours du voyage intérieur et l'appartenance de cette démarche au romantisme est le deuxième parage qu'explore notre volume: Henri Bonnet interroge l'ordonnancement secret des "Lettres " et le discours mystique qu'il peut convoquer ; Nigel Harkness suit, dans la sinuosité du volume, le fil d'une quête d'identité ; José-Luis Diaz décrit les règles de " George Sand et compagnie ", le jeu de rôles sérieux grâce auquel le Voyageur trouve son chemin parmi les autres; Pierre Laforgue situe les Lettres d'un voyageur dans leur contexte de publication (I837) pour apprécier leur rapport avec le romantisme.

Une troisième série d'études se consacre au discours sur les arts qui, réitéré sous différentes formes tout au long du recueil, constitue celui-ci en un traité d'esthétique. Nicole Mozet fait l'inventaire critique des figurations de soi en artiste qu'essaie le Voyageur ; Olivier Bara repense, à partir de l'exemple du discours sur l'opéra, les liens qui unissent, chez Sand, esthétique, éthique et politique ; Suzel Esquier analyse la place de la musique, idée, valeur et sensation, dans l'expérience du Voyageur ; Yvon Le Scanff expertise l'art pictural dont relèvent les séquences de description de paysages ; Nicole Savy reconstitue, à partir du cas particulier de la sculpture, l'ensemble du discours de l'auteur sur les rapports entre art et nature.

Ces réflexions ont trouvé l'occasion de se formuler grâce aux journées d'étude qui, les I6 et I7 juin 2004, se sont tenues à l'université Stendhal. Le Centre d'études stendhaliennes et romantiques, composante de l'équipe Traverses XIX-XXI, a organisé l'événement avec la collaboration de l'équipe XIX siècle de l'UMR LIRE - Lyon 2 : je souhaite remercier les responsables de ces unités de recherche, Marie-Rose Corredor, Chantal Massol et Christine Planté, pour avoir rendu ces travaux possibles. Je voudrais également remercier Catherine Mariette-Clot pour son aide lors de ces journées, ainsi que les 
différentes personnes qui y ont apporté leur concours par des interventions précieuses, en particulier Jean-Hervé Donnard, Michel Lafon et Shira Malkin.

Ces journées prenaient place dans le contexte de l'année du bicentenaire de la naissance de George Sand : puisque commémoration nationale il y avait, Simone Vierne a souhaité donner à celle-ci une inflexion locale en rappelant, par une allocution d'ouverture, la part éminente qu'avaient eue des Grenoblois (chercheurs de l'Université et, plus largement, amoureux de la littérature de l'agglomération grenobloise) dans la relance des études sur George Sand dans la deuxième moitié du XXe siècle. Ce propos est reproduit ici à la place liminaire qui était la sienne lors des journées de juin 2004.

Enfin, qu'il me soit permis de dire un mot de la dédicace qui ouvre ce volume : Henri Bonnet mérite bien un hommage marqué de la part de tous ceux qui sont attachés aux Lettres d'un voyageur. Depuis l'introduction qu'il a donnée en I97I pour son édition du recueil, jusqu'à sa contribution dans le présent volume, il s'est montré un lecteur exemplaire de cet ouvrage pourtant rétif à se laisser cerner par le discours : communicatif de ses intuitions, généreux dans ses interprétations et exigeant dans leur formulation, il est le guide qu'on est heureux d'avoir rencontré sur la trace du Voyageur. 